\title{
CD19CAR-CD3zeta-expressing Autologous T lymphocytes
}

National Cancer Institute

\section{Source}

National Cancer Institute. CD19CAR-CD3zeta-expressing Autologous T lymphocytes. NCI Thesaurus. Code C88266.

Autologous T-lymphocytes transduced with a modified lentiviral vector expressing a chimeric antigen receptor (CAR) consisting of an anti-CD19 scFv (sing le chain variable fragment) and the zeta chain of the TCR/CD3 complex (CD3-zeta), with potential immunomodulating and antineoplastic activities. Upon transfusion, CD19CAR-CD3zetaexpressing autologous T-lymphocytes are directed to CD19-expressing tumor cells, thereby inducing a selective toxicity only in these tumor cells. The CD19 antigen is a B-cell specific cell surface antigen expressed in all B-cell lineage malignancies. CD3-zeta (or CD247) is a transmembrane signaling adaptor polypeptide that regulates the assembly of complete T-cell receptor complexes and their expression on the cell surface. 